\title{
Responding in the presence of free food: Differential exposure to the reinforcement source
}

\author{
PETER MITCHELL and K. GEOFFREY WHITE \\ Victoria University, Wellington, New Zealand
}

\begin{abstract}
Preference in rats for response-contingent reinforcement vs free food was studied as a function of differential exposure to the response-contingent and free reinforcement sources. Subjects trained primarily with response-contingent reinforcement exhibited responding for food in the presence of free food in later preference tests, and allocated more time to the response-contingent reinforcement source than did subjects trained with equal exposure to the reinforcement sources. Subjects trained to take freely available food did not respond in the presence of free food. The phenomenon of responding for food in the presence of free food was thereby demonstrated to be a consequence of prior operant or instrumental training.
\end{abstract}

Responding for food in the presence of free food is a phenomenon that has aroused considerable recent interest because it contradicts the common assumption that an animal prefers freely available food to earning food by performing some instrumental or operant response. The typical procedure involves first training a hungry rat to press a bar for food pellets. Then, in a subsequent preference test, the animal is given a choice between barpressing for food pellets or obtaining identical food pellets from a cup in the experimental chamber. The result of a number of studies is that animals prefer to respond for a reinforcer although an identical reinforcer is freely available (Jensen, 1963; Neuringer, 1969, 1970a; Tarte \& Snyder, 1973).

The preference for earned vs free reinforcement has commonly been labeled "contrafreeloading," but the designation is unfortunate in that it implies a certain theoretical stance. Indeed, it is our contention that "freeloading" is not necessarily what one might expect of an animal that has been trained to earn its food.

There have been two main explanations for the phenomenon of responding in the presence of free food, neither of which is satisfactory. One account appeals to the "intrinsic attractiveness" of responding for food (Jensen, 1963) and includes the speculative notion of a "work ethic" in animals (Singh \& Query, 1971). The other attributes responding in the presence of free food to the elicitation of species-specific behaviors related to the consummatory response (Powell, 1974).

This report is based on an MA thesis submitted to the Victoria University of Wellington by the first author. Carol Logie and Melva Maguire assisted in the preparation of the manuscript. Reprints may be obtained from Dr. K. G. White, Department of Psychology, Victoria University of Wellington, Wellington, New Zealand. This paper is sponsored by Professor M. R. D'Amato, who takes full editorial responsibility.
The "intrinsic appeal" explanation of responding in the presence of free food assumes that the act of performing the response is reinforcing in itself. Therefore, a general prediction is that the more prior instrumental training an animal receives, the greater the tendency to respond in the presence of free food. This prediction was confirmed in Jensen's (1963) seminal experiment, where rats in each of six groups made $40,80,160,320,640$, or 1,280 continuously reinforced barpresses during prior training. In the single test session, the animal could choose between barpressing for food pellets or obtaining identical pellets from a cup in the experimental chamber. Jensen found an exponential relation between the number of pellets earned by responding and the number of responses in prior training. But in later studies, where rats could either run an alley for one pellet in the goalbox or eat pellets freely available in the startbox, the reverse was the case; animals with the greater amount of prior training in the runway took more pellets freely than those with less training (Jensen, Leung, \& Hess, 1970; Leung, Jensen, \& Tapley, 1968).

The main argument against the assumption that barpressing has intrinsic appeal is that, if this was so, the animal should continue to respond in experimental extinction. This is not the case, as Duncan and Hughes (1972) and Neuringer (1969) have shown. Furthermore, to argue that the high probability of response observed in the presence of free food is due to the intrinsic high probability of the response (cf. Sawisch \& Denny, 1973 ) is patently tautologous and does not specify the conditions determining the probability of the response. Intrinsic appeal thus takes on the guise of an explanatory fiction and, in any case, is contradicted by the fact that responding in the presence of free food declines in experimental extinction.

Carder (1972) and Powell (1974) have attributed responding in the presence of free food to the elicitation 
of food-directed behaviors as part of the innately determined consummatory pattern. Keypecking in both crows and pigeons, for example, mimics the consummatory behavior of the animal and is engendered by food presentation (Powell, 1974). Treadle-pressing in pigeons is not, however, determined by innate factors (Green \& Rachlin, 1975), although the treadle-pressing response persists in the presence of free food (Neuringer, 1969). Moreover, a species variable is unlikely to be responsible for the phenomenon since it occurs for a variety of species, such as domestic fowls, cats, mongolian gerbils, pigeons, crows, and children (Duncan \& Hughes, 1972; Koffer \& Coulson, 1971; Lambe \& Guy, 1973), and reinforcers (Knutson \& Carlson, 1973; Robertson \& Anderson, 1975; Tarte, Townsend, Vernon, \& Rovner, 1974). Responding in the presence of free food therefore cannot be accounted for in terms of the animal's species-specific consummatory or fooddirected behavior.

The purpose of the present paper was to present evidence for an alternative interpretation. Specifically, that responding occurs in the presence of free food depends upon the fact that the animal has been trained to respond for food prior to preference testing. That is, the "diligent" behavior of responding for food in the presence of free food is simply the result of the animal's previous conditioning history. The hypothesis was suggested by the fairly consistent result that the number of responses made in the test for preference (between response-contingent and free food) is related to the number of pellets earned or the number of operant responses emitted in prior training (Jensen, 1963; Jensen, Leung, \& Hess, 1970; Stoltz \& Lott, 1964; Tarte $\&$ Snyder, 1973). Indeed, if the amount of prior training is an important variable, then it is not surprising that many studies have demonstrated a preference for earned vs free reinforcement in that most studies have administered a far greater amount of earned than free training.

The "conditioned diligence" hypothesis was tested directly by training one group of subjects to respond for food and another to receive food freely. A third group was exposed to both noncontingent (free) food presentations and response-contingent reinforcement equally often. The first group corresponded to the groups exhibiting responding in the presence of free food of the previous studies, while the second group, which predominantly received free food, was crucial to testing the "conditioned diligence" hypothesis. According to this hypothesis, the free-food group should exhibit a strong preference for freely available food, while, according to both "intrinsic appeal" and "consummatory response" interpretations, the free-food group was expected to exhibit a preference for response-contingent reinforcement.

The important independent variable in the present experiment was frequency of presentation of noncontingent (free) and response-contingent (earned) reinforcement. Therefore, it was necessary to specify exactly the frequency of occurrence of the free food. Many previous studies have not specified the rate or frequency of free-food presentation in that reinforcers were presented en masse, as in the case of a cup of food pellets. In the present experiment the programmed rate of both free and response-contingent reinforcement during training and testing was the same, whereas total frequency of reinforcement was manipulated by varying the number of training sessions with each reinforcement source.

Definition of a preference index was a further problem which previous studies have not coped with satisfactorily. For example, some studies have recorded the amount of reinforcement (or number of food pellets) obtained from both sources and have calculated the percentage of pellets earned (Hothersall, Huey, \& Thatcher, 1973; Osborne \& Shelby, 1975; Tarte \& Snyder, 1973). Others have recorded only the number of responses (Alferink, Crossman, \& Cheney, 1973), while yet others have measured just the absolute amount of free food consumed without recording response frequency (Taylor, 1975). Still others have measured the time required to stop eating free food and perform the response (Jensen, Leung, \& Hess, 1970) or the time to stop responding and eat free food (Koffer \& Coulson, 1971). None of these measures is satisfactory since preference is confounded with the differential effects of the behaviors involved in responding or consuming free food. In the present experiments, the time allocated to each of the two alternative reinforcement sources provided an index of preference which was not confounded with the effects of the different responses. Baum and Rachlin (1969) and Premack (1965) have shown that the allocation of time to different activities is a sensitive and reliable index of preference.

\section{METHOD}

\section{Subjects}

Twelve experimentally naive female hooded rats between 70 and 120 days old at the beginning of the study were used as subjects. Subjects were individually housed and maintained at $80 \%( \pm 10 \%)$ of their free-feeding body weights throughout the experiment.

\section{Apparatus}

Subjects were trained and tested in a modified Lehigh Valley Electronics experimental chamber (Model 143-21). The modification involved the addition of a second identical liquid dipper to the opposite side of the chamber from the existing dipper. Thus, there was a dipper, retractable response lever, and stimulus lights on the right-hand side of the chamber, and a dipper and stimulus lights on the left-hand side. A force of $.024 \mathrm{~N}$ was required to operate the lever. Effective leverpresses produced an audible feedback click. The grid floor was wired such that the amount of time allocated to each half of the chamber could be measured. The reinforcer was a sweetened condensed milk solution mixed one part milk to one part water. Each dipper, when filled, held $.01 \mathrm{cc}$ of condensed milk. Masking noise was provided, and general illumination was produced by a standard houselight. Sound was attenuated by enclosing the chamber in a box lined with pinex ceiling tiles, and a portion of one side was 
removed for insertion of the subject and for observation. Controlling and recording equipment was located in an adjacent room.

\section{Procedure}

The procedure involved three stages: preliminary training, training, and testing. Sessions were conducted daily at all stages of the experiment.

Preliminary training. During preliminary training all rats were allowed to make 100 continuously reinforced leverpress responses on the right side of the chamber during one session. The response was acquired without shaping and was emitted at an average rate of one per $10 \mathrm{sec}$. During another session all rats received 100 dipper presentations from the left dipper delivered independently of the subjects' behavior according to a variable-time (VT) 10-sec schedule. The order of the first two sessions was balanced across subjects. Subjects were then matched and assigned to one of the three groups on the basis of the rate of leverpressing during preliminary training.

Training. Training consisted of 10 daily sessions on either a variable-interval (VI) 5 -sec or a VT 5-sec schedule of reinforcement, with sessions terminating after 100 reinforcements. During all stages of the experiment, a white stimulus light above the lever on the right side and another in a similar position on the left side indicated whether the VI or VT schedule was in effect. The tapes that programmed the intervals for the two schedules were identical and were constructed from an arithmetic progression described by Catania and Reynolds (1968). The VT schedule delivered a programmed reinforcer regardless of the animal's behavior or position in the chamber. The lever was retracted during VT. The VI schedule delivered a reinforcer contingent upon occurrence of the first leverpress after the prescribed interval. The amount of VI or VT training was different for different groups. The four subjects in Group 1 received a total of 500 response-dependent (VI) and 500 response-independent (VT) reinforcers over the 10 days. A day of VI was alternated with a day of VT. The four subjects in Group 2 received 1,000 responsedependent reinforcers only, and the four subjects in Group 3 received 1,000 response-independent reinforcers only. Thus, by the end of training (including preliminary training), subjects in Group 1 had received a total of 600 response-dependent and 600 response-independent reinforcers. Group 2 had received 1,100 response-dependent and 100 response-independent reinforcers, and Group 3 had received 100 response-dependent and 1,100 response-independent reinforcers.

Testing. During each of three test sessions, both stimulus lights were on and the VI 5-sec and VT 5-sec schedules were in effect concurrently. Each session was of 20-min duration. During all training and testing sessions, time spent on each side, total time, number of dipper presentations, and number of leverpresses were recorded.

\section{RESULTS}

Table 1 presents response frequencies and time allocated to the VT and VI reinforcement sources per session, averaged over the three test sessions for each subject. Response rates were calculated for total session time as the time base. Neither response rates nor time allocated varied significantly over the three test sessions. The subjects in Group 1 allocated an average of $30 \%$ of thieir time to the VI reinforcement source and responded at mean rate of 7.8 responses $/ \mathrm{min}$. The subjects in Group 2 trained primarily with the VI source allocated an average of $60 \%$ of their time to the VI source and responded at a mean rate of 23.8 responses/ min. The subjects in Group 3 trained primarily with
Table 1

Time Allocated (Seconds) to the VI and VT Reinforcement Sources and Responses Emitted Per Session Averaged Over Three Test Sessions

\begin{tabular}{rccc}
\hline Subject & Time in VI & Time in VT & $\begin{array}{c}\text { Total } \\
\text { Responses }\end{array}$ \\
& Group 1 & $(600$ VI, 600 VT Reinforcements) \\
7 & 346 & 625 & 184 \\
8 & 280 & 669 & 118 \\
12 & 196 & 780 & 110 \\
17 & 342 & 607 & 213 \\
& Group 2 (1100 VI, 100 VT Reinforcements) \\
11 & 572 & 373 & 500 \\
6 & 758 & 235 & 665 \\
14 & 513 & 430 & 365 \\
16 & 446 & 487 & 373 \\
& Group 3 (100 VI, 1100 VT Reinforcements) \\
3 & 32 & 946 & 0 \\
4 & 59 & 845 & 0 \\
10 & 20 & 1062 & 0 \\
15 & 31 & 951 & 4 \\
\hline
\end{tabular}

the VT source allocated an average of just $4 \%$ of their time to the VI source and emitted an average of .05 responses/min. Differences between the groups were highly significant for both relative time allocation $[F(2,9)=44.79, p<.001]$ and response rate $[F(2,9)=$ $31.47, \mathrm{p}<.001]$. The highly significant productmoment correlation $(\mathrm{r}=.975)$ between response rate and time allocation data confirms the reliability of time allocation as an index of preference.

\section{DISCUSSION}

The present experiment demonstrated that animals trained to barpress with response-contingent VI reinforcement (Group 2) continued to emit a high response rate in the presence of noncontingent (free) reinforcement delivered according to a VT schedule. This particular result thereby replicates the previous findings of responding in the presence of free food (Carder \& Berkowitz, 1970; Neuringer, 1970a; Tarte \& Snyder, 1973). Furthermore, that the subjects of Group 2 preferred the earned (VI) source to the free source of reinforcement is indicated by the fact that greater than $50 \%$ of their time was allocated to the VI reinforcement source.

The subjects in Group 1 exposed equally to VI and VT reinforcement in prior training also continued to respond in the presence of free food, but at a lower rate than exhibited by the Group 2 subjects. Amount of training with VI relative to VT reinforcement thus determines the extent to which responding in the presence of free food is observed.

The important result was that animals exposed to a far greater frequency of noncontingent (free) than VI reinforcement (Group 3) showed a clear preference for free reinforcement and generally did not respond in the presence of free food during testing. Three subjects in Group 3 did not respond in any test session, while one emitted an average of 4 responses/20-min session. The result confirms the conditioned diligence hypothesis, that animals trained to respond in prior operant or instrumental training will continue to respond in the presence of free food during subsequent testing; animals exposed to free reinforcement during prior training will freeload or continue to take freely available food in preference to responding for food in the preference test. 
The data clearly contradict the notions that responding in the presence of free food is a consequence of the "intrinsic appeal" of the response or of food-directed behavior patterns, since both explanations predict that the Group 3 animals would exhibit a moderately high response rate in the preference test. It is also unlikely that the present data for Group 3 can be accounted for by superstitious behavior which might have interfered with responding, for the following reasons. First, the highest probability superstitious response was likely to have been barpressing since initial response-contingent reinforcement is sufficient for subsequent response maintenance by VT reinforcement (Neuringer, 1970b). Second, superstitious behavior is unlikely to occur in the context of VT reinforcement and, further, was not observed during occasional observation by the experimenters.

The present data clearly support the following conclusion. Responding in the presence of free food (the so-called "contrafreeloading" phenomenon) is the product of the animal's previous conditioning history in that the animal has been trained to respond for food rather than to take freely available food before the preference test is conducted. If the animal is trained to take freely available food, then responding in the presence of free food is not observed. Instead, the animal exhibits a preference for free food (or freeloading). The preference for responsecontingent reinforcement observed in previous studies is therefore seen to be the result of considerable prior training with response-contingent reinforcement rather than with free food.

\section{REFERENCES}

Alferink, L. A., Crossman, E. 'K., \& Cheney, C. D. Control of responding by a conditioned reinforcer in the presence of free food. Animal Learning \& Behavior, 1973, 1, 38-40.

BaUm, W. M., \& RACHLIN, H. C. Choice as time allocation. Journal of the Experimental Analysis of Behavior, 1969, 12, 861-874.

CARDER, B. Rats' preference for earned in comparison with free liquid reinforcers. Psychonomic Science, 1972, 26, 25-26.

CARder, B., \& Berkowitz, K. Preference for earned in relation to free food. Science, 1970, 167, 1273-1274.

Catania, A. C., \& Reynolds, G. S. A quantitative analysis of the responding maintained by interval schedules of reinforcement. Journal of the Experimental Analysis of Behavior, 1968, 11, 327-383.

Duncan, J. H., \& Hughes, B. O. Free and operant feeding in domestic fowls. Animal Behavior, 1972, 20, 775-777.

GreEN, L., \& RACHLIN, H. Economic and biological influences on a pigeon's key peck. Journal of the Experimental Analysis of Behavior, 1975, 23, 55-62.

Hothersall, D., Huey, D., \& Thatcher, K. The preference of rats for free or response-produced food. Animal Learning \& Behavior, 1973, 1, 241-243.

Jensen, G. D. Preference for bar pressing over "freeloading" as a function of number of rewarded presses. Journal of Experimental Psychology, 1963, 65, 451-454.

Jensen, G. D., Leung, C. M., \& Hess, D. T. "Freeloading" in the Skinner box contrasted with freeloading in the runway. Psychological Reports, 1970, 27, 67-73.

Knutson, J. F., \& Carlson, C. W. Operant responding with free access to the reinforcer: $A$ replication and extension. Animal Learning \& Behavior, 1973, 1, 133-136.

Koffer, K., \& Coulson, G. Feline indolence: Cats prefer free to response-produced food. Psychonomic Science, $1971,24,41-42$.

LAmbe, D. R., \& GuY, E. G. Comparison of the preference for free vs. earned food in rats and mongolian gerbils. Proceedings of the Ohio Academy of Science, April 1973.

Leung, C. M., Jensen, G. D., \& TAPley, R. P. "Freeloading" in a runway as a function of amount of training and type of reinforcement schedule. Psychological Reports, 1968, 22, 211-214.

Neuringer, A. J. Animals respond for food in the presence of free food. Science, 1969, 166, 399-400.

Neuringer, A. J. Many responses per food reward with free food present. Science, 1970, 168, 503-504. (a)

Neuringer, A. J. Superstitious key pecking after three peck-produced reinforcements. Journal of the Experimental Analysis of Behavior, 1970, 13, 127-134. (b)

Osborne, S. R., \& Shelby, M. Stimulus change as a factor in response maintenance with free food available. Journal of the Experimental Analysis of Behavior, 1975, 24, 17-21.

Powell, R. W. Comparative studies of the preference for free vs. response-produced reinforcers. Animal Learning \& Behavior, 1974, 2, 185-188.

Premack, D. Reinforcement theory. In D. Levine (Ed.), Nebraska Symposium on Motivation (Vol. 13). Lincoln: University of Nebraska Press, 1965. Pp. 123-180.

Robertson, L. C., \& ANDERson, S. C. The effects of differing type and magnitude of reward on the contrafreeloading phenomenon in rats. Animal Learning \& Behavior, 1975, 3, 325-328.

Sawisch, L. P., \& Denny, M. R. Reversing the reinforcement contingencies of eating and keypecking behaviors. Animal Learning \& Behavior, 1973, 1, 189-192.

Singh, D., \& QUERY, W. T. Preference for work over "freeloading" in children. Psychonomic Science, 1971, 24, 77-79.

StolTz, S. B., \& LoTT, D. F. Establishment in rats of a persistent response producing a net loss of reinforcement. Journal of Comparative and Physiological Psychology, 1964, 57, 147-149.

TARTE, R. D., \& SNYDER, R. L. Some sources of variation in the barpressing versus freeloading phenomenon in rats. Journal of Comparative and Physiological Psychology, 1973, 84, 128-133.

Tarte, R. D., Townsend, S. G., Vernon, C. R., \& Rovner, L. An examination of various deprivationreward combinations in the barpressing vs. freeloading phenomenon in rats. Bulletin of the Psychonomic Society, 1974, 3, 227-229.

TAYLOR, G. Discriminability and the contrafreeloading phenomenon. Journal of Comparative and Physiological Psychology, 1975, 88, 104-109.

(Received for publication November 2, 1976; revision received March 25, 1977.) 